\title{
Cognitive impairment among patients with diabetes in Saudi Arabia: a cross-sectional study
}

\author{
Rania Naguib ${ }^{1,2^{*}}$ (D, Eman S. Soliman ${ }^{1,3}$, Fatima Mohammed Neimatallah', Najd Sulaiman AlKhudhairy', \\ Amjad Muashaq ALGhamdi', Reema Saad Almosa', Kholud Abdullaziz Aldashash', \\ Bashayer Yaqoub Alkhalifah ${ }^{1}$ and Hala Elmorshedy ${ }^{1,4}$
}

\begin{abstract}
Background: The incidence of cognitive impairment $(\mathrm{Cl})$ is higher in patients with diabetes mellitus (DM). The association between $\mathrm{DM}$ and $\mathrm{Cl}$ and the risk factors for $\mathrm{Cl}$ need to be addressed to estimate the prevalence of cognitive impairment in patients with DM and to identify the potential risk factors. The study is a cross-sectional study using a convenient sample of 269 subjects. Sociodemographic diabetes-related variables including biochemical markers were collected. $\mathrm{Cl}$ and diabetes-related distress (DRD) were assessed using the Arabic version of Montreal Cognitive Assessment scale (MoCA) and the Diabetes Distress Screening Scale respectively.

Results: Overall, $80.3 \%$ had cognitive impairment while 33.8\% had severe impairment. Older age, female gender, low level of education, and low income were associated with Cl; duration of diabetes and DRD were associated with $\mathrm{Cl}$ while ophthalmic complications were associated with severe $\mathrm{Cl}$. Duration of diabetes was inversely associated with $\mathrm{Cl}$. Level of $\mathrm{HbA} 1 \mathrm{C}$ was significantly higher in patients with severe $\mathrm{Cl}$, and the probability of $\mathrm{Cl}$ increased as the level of $\mathrm{HbA1c}$ increased. Low level of education was associated with severe $\mathrm{Cl}$, and $\mathrm{Cl}$ was two times more likely among patients with DRD.

Conclusion: $\mathrm{Cl}$ was higher than worldwide figures. Elderly females with low educational level, long duration of DM, and low socioeconomic status are at more risk. The probability of severe $\mathrm{Cl}$ increased with increased level of $\mathrm{HbA1c}$. Screening for $\mathrm{Cl}$ in patients with diabetes along with intervention programs while considering the DRD and the level of $\mathrm{HbA} 1 \mathrm{c}$ is crucial.
\end{abstract}

Keywords: Cognitive impairment, Diabetes mellitus, Glycated hemoglobin, MoCA, Diabetes-related distress, Saudi Arabia

\section{Background}

Diabetes mellitus is a chronic metabolic disease that is characterized by hyperglycemia which affects most of the body systems. Diabetic patients usually develop diverse microvascular, macrovascular, and neuropathic

\footnotetext{
* Correspondence: Ranianaguib2000@yahoo.com

'Clinical Science Department, College of Medicine, Princess Nourah bint Abdulrahman University, Riyadh, Kingdom of Saudi Arabia

${ }^{2}$ Internal Medicine Department, Alexandria Faculty of Medicine, Alexandria University, Alexandria, Egypt

Full list of author information is available at the end of the article
}

complications making diabetes a major health problem [1]. Several studies have linked cognitive impairment with diabetes mellitus [2-4]. More recent studies showed that the incidence of cognitive impairment is higher in patients with diabetes mellitus compared to general population $[5,6]$ and that diabetes mellitus is considered as a risk factor for cognitive impairment [7]. Several studies reported an association between high blood glucose levels and the onset and progression of cognitive impairment [8-11]. It was also reported that

\section{Springer Open}

(๑) The Author(s). 2020 Open Access This article is licensed under a Creative Commons Attribution 4.0 International License, which permits use, sharing, adaptation, distribution and reproduction in any medium or format, as long as you give appropriate credit to the original author(s) and the source, provide a link to the Creative Commons licence, and indicate if changes were made. The images or other third party material in this article are included in the article's Creative Commons licence, unless indicated otherwise in a credit line to the material. If material is not included in the article's Creative Commons licence and your intended use is not permitted by statutory regulation or exceeds the permitted use, you will need to obtain permission directly from the copyright holder. To view a copy of this licence, visit http://creativecommons.org/licenses/by/4.0/. 
less than $4 \%$ of the healthy subjects aged 65 years or less are suffering from cognitive impairment [12].

Cognitive impairment is defined as difficulty remembering, learning new things, concentrating, or making decisions that affect everyday life [1]. It is a major community concern because it results in functional disability, and it increases healthcare costs [13]. It might adversely affect the management plan or cause serious harm specially in patients with diabetes, since patients' concepts regarding the need for regular follow-up, self-care, adherence to diet, exercise, and medications depend mainly on an intact memory $[1,14]$. Patients with cognitive impairment might be at risk of having complications from treatment like developing hypoglycemia because of either omission of meals or wrong dose or timing of insulin or oral medications $[1,15]$.

The exact mechanism for the development of cognitive impairment in diabetes mellitus is complex. One study suggests cerebrovascular disease as a risk factor [13]. Other studies suggest that fluctuation of blood glucose level, end products of metabolism, insulin resistance, concomitant hypertension, amyloidosis, depression, and some physical and psychological factors may play significant roles [2, 16]. Chronic hyperglycemia has been proven to be toxic to the neurons due to the production of advanced glycation end products causing oxidative damage which causes neuronal injury $[8,17]$. Microangiopathy, oxidative stress, inflammation, and dyslipidemia are other important factors which can lead to neuronal damage resulting in cognitive impairment [1]. Other suggested mechanisms for cognitive impairment among individuals with diabetes mellitus include expression of insulin-degrading enzyme and occurrence of severe hypoglycemic events [18]. The duration of diabetes and poor glycemic control has been always considered as important contributors to the impact of diabetes mellitus on cognitive impairment $[7,19]$. The longer the duration of diabetes mellitus, the more likely is the occurrence of complications, including cognitive compromise [19].

Some studies assessing the prevalence of cognitive impairment in the Middle East and Arabic-speaking countries demonstrated that the range of cognitive impairment in this region ranges from 4.4 to $32 \%$ [20, 21]. A recent study assessing the prevalence of cognitive impairment among Saudi population aged 60 years and above demonstrated a prevalence of $46 \%$ [21]. It is still controversial to determine which domain of cognition is affected the most in patient with diabetes [13].

Diabetes-related distress (DRD) is defined as significant psychosocial factors that have been demonstrated to impact diabetes outcomes. Diabetes distress depicts the logical and situational challenges which an individual can encounter concerning the management, support, and emotional stress of diabetes. Several studies demonstrated that diabetes-related distress affects glycemic control, supporting the impact of diabetes distress on diabetes outcomes [22]. In spite of the obvious relation between diabetes and DRD, the relation between cognitive impairment and DRD needs to be addressed.

Although diabetic patients are at increased risk to develop cognitive impairment, the cognitive function of patients with diabetes is not usually assessed in routine clinical care. There are no data available on the prevalence of cognitive impairment in the diabetic Saudi population. It is crucial to understand the association between cognitive impairment and diabetes mellitus in order to maximize the quality of life. The aim of this study is to estimate the prevalence of cognitive impairment in patients with DM and to identify the potential risk factors.

\section{Methods \\ Study design}

We conducted a cross-sectional study using a convenient sample of 269 diabetic subjects recruited from governmental and university general tertiary care hospitals in Riyadh, Saudi Arabia, from September 2019 till March 2020. Subjects of both genders aged 18 years and above diagnosed with type 1 or type 2 diabetes for at least 2 years whose diagnosis was based on laboratory tests (HbA1c, fasting plasma glucose, and 2-h post-prandial plasma glucose) and are receiving any modality of treatment for diabetes (diet, exercise, oral hypoglycemic medications, and/or insulin) were included in this study. Patients with an advanced comorbid medical condition that could have affected cognitive function such as developmental disorders, mental retardation, advanced neurological condition, uncontrolled psychiatric problems, dementia, advanced cardiac or pulmonary conditions, severe liver and/or renal failure, advanced rheumatological disorder, severe anemia, using steroids for the treatment of any disease, and patients with speech or hearing disabilities were excluded.

\section{Sample size calculation}

Sample size was calculated using STATA Ver.16 (Stata Corp, College Station, TX, USA), assuming prevalence of cognitive impairment at $0.6 \pm 0.1, P<0.05$, and power of study 0.8; the minimal sample size was 197, we increased the sample size to 269 to compensate for missing data.

Ethical considerations The study was conducted in accordance with the Helsinki Declaration and after the ethical approval of the Institutional Review Board at Princess Nourah bint Abdulrahman University, Riyadh, KSA (IRB-PNU:19-0139), date of approval: 21 October 2019. Informed verbal consent was taken from all study 
participants. Verbal consent was approved by the ethics committee. The IRB recommends the use of verbal consent in survey procedures unless information is obtained in such a manner that human subjects can be identified or subjects' responses outside the research could place them at risk of criminal or civil liability or may damage their employability, or reputation.

\section{Data collection method}

\section{Socio-demographic and diabetes-related variables}

This part was developed after searching the literature for similar studies $[1,16]$. Additional questions were devised de novo specifically for this survey to evaluate the sociodemographic profile and contributing factors for cognitive impairment in the studied sample. It was first developed in English and was translated into Arabic by two bilingual speakers. It was revised by two public health experts for face validity and one field expert for content validity. It included multiple choice questions inquiring about the socio-demographic variables like age, gender, marital status, education, job, and monthly income, details about patients' disease such as duration of diabetes and if the subject suffers from any complications related to diabetes like numbness and self-reported ophthalmic complications. Data were collected from the patients' medical records documenting the body mass index (BMI) (calculated as weight divided by height square), lipid profile (cutoff value according to the manufacturer), and associated hepatic, renal, cardiac, and neurological co-morbidities. Hemoglobin A1c (HbA1c) level which reflects the glycemic control status of approximately the previous 3 months was recorded. A HbA1c percentage of $6.5 \%$ was considered the cut point for diagnosis of diabetes $[8,23]$. Blood pressure (BP) was recorded. Patients with persistent $\mathrm{BP}$ readings more than 140/90 were considered uncontrolled [24] and were excluded from the study.

\section{Measurement scales \\ Cognitive impairment}

We evaluated the cognitive impairment using the Arabic version of the Montreal Cognitive Assessment (MoCA) (version 7.1 original version) after getting authors' permission. The scale assessed seven cognitive domains, namely, visuospatial/executive function, naming, attention, language, abstraction, delayed recall, and orientation [25]. The possible scores ranged from 0 to 30 with higher scores indicating better cognitive status; the score was adjusted by adding one point, if years of education are $\leq 12$ years. The cutoff of cognitive impairment was $<26$ [26]. A cutoff value of $<20$ was used to determine the prevalence of severe cognitive impairment $[27,28]$ among the Saudi population and to identify the possible risk factors associated with it. Medical students were trained by one of the authors-a psychiatrist specialist-to measure the cognitive impairment using the MOCA scale. In this study, the Cronbach's alpha $\alpha$ was 0.76 , suggesting a good internal consistency.

\section{Diabetes-related distress}

To measure the diabetes-related distress (DRD), we used the Arabic version of DDS-17 [29]. This scale assesses DRD during the past month; it includes 17 items, each item is measured on a six-point Likert scale: a mild to moderate problem is 1 or 2 , a moderate to serious problem is 3 or 4 , and a serious problem is 5 or 6 . The total score of DDS-17 was computed by summing the 17 items and dividing by 17. The cutoff for clinically significant DRD is $>2$ [30]. The internal consistency of the DDS-17 for this study was excellent, the Cronbach's alpha $\alpha$ was 0.92 .

The questionnaire was pilot tested on 30 subjects to ensure both comprehension and readability and to estimate the time required for data collection. Six trained medical students were responsible for obtaining verbal consent from participants, explaining the purpose of the study, distributing the questionnaire, and ensuring that all questions are answered. If any question found to be redundant or inappropriate during the pilot test, it was modified, and a final questionnaire was prepared.

\section{Statistical analyses}

We used STATA Ver.16 (Stata Corp, College Station, TX, USA) for data analysis. Quantitative variables were described as means $\pm \mathrm{SD}$, or as median with interquartile range (IQR) if variables were not normally distributed, while qualitative variables were described as frequency and percentages with CI 95\%. Difference in the mean total score and in the subdomain scores of MoCA in relation to duration of diabetes was tested by one-way ANOVA and LSD post hoc analyses, while association between the level of cognitive impairment and socio-demographic and diabetes-related factors was evaluated by Chi-square test and Cramer's V to assess the strength of association. We conducted logistic regression analyses to explore the association between the level of cognitive impairment and diabetes-related factors including, duration, control of diabetes according to the level of $\mathrm{HbA1c}$, and comorbidities which showed significant association in bivariate analysis, and controlled for socio-demographic variables. The level of statistically significant results was set at $P<0.05$. We estimated the predicted probability of cognitive impairment in relation to the level of HbA1c using the marginal effect commands of STATA 16. 


\section{Results}

In total, the study included 269 participants, more than $90 \%$ of them aged $\geq 40$-years old while the mean age was 56 years. Overall, about one-fifth was single, and females accounted for nearly two-third of the study population. Holders of university degree amounted to $38.7 \%$, and $60 \%$ were not employed-including housewives and retired individuals-nevertheless income was considered enough by most of participants. More than $90 \%$ of participants were overweight or obese (Table 1). As much as $87.4 \%$ had type 2 diabetes. According to the level of HbA1c, diabetes was controlled in less than one-third of patients, and the duration of diabetes was $\geq 10$ years in more than half of the sample. Self-reported comorbidities exceeded $50 \%$ each for ophthalmic problems and symptoms of peripheral neuritis peculiar to diabetes. Review of hospital records revealed hepatic comorbidities in more than one-fifth of patients followed by renal and cardiac problems each in more than $10 \%$ while the neurological problems were the least of all comorbidities. Triglycerides and LDL-C were high in $26.2 \%$ and $44.2 \%$ respectively while as much as $85 \%$ had low levels of HDL-C. Of note, the study revealed diabetic-related distress in more than one-third of patients (Table 2).

Table 1 Sociodemographic characteristics of patients with diabetes, $n=269$

\begin{tabular}{ll}
\hline Characteristics & $\boldsymbol{N}(\%) /$ mean \pm SD \\
\hline Age in years, mean \pm SD & $56.01 \pm 9.97$ \\
Age categories & $18(6.7)$ \\
$\quad<40$ years old & $137(50.9)$ \\
$40-59$ years old & $114(42.4)$ \\
$\geq 60$ years old & \\
Gender & $185(68.8)$ \\
Females & $84(31.2)$ \\
Males & \\
Social status & $208(77.3)$ \\
Married & $61(22.7)$ \\
Single & \\
Education & $165(61.3)$ \\
Below bachelor & $104(38.7)$ \\
Bachelor & \\
Occupation & $187(69.5)$ \\
Unemployed & $82(30.5)$ \\
Employed & \\
Income & $28(10.4)$ \\
Enough and can save & $207(77.0)$ \\
Enough & $34(12.6)$ \\
Not enough & \\
\hline
\end{tabular}

Table 2 Clinical and biochemical profiles of patients with diabetes

\begin{tabular}{|c|c|}
\hline Characteristics & $N(\%) /$ median (IQR) \\
\hline \multicolumn{2}{|l|}{ *BMI (weight in $\mathrm{kg} /$ height in $\mathrm{m}^{2 \text { ) }}$} \\
\hline$<18.5-24.9$ & $12(4.6)$ \\
\hline $25-29.9$ & $64(24.4)$ \\
\hline$\geq 30$ & $186(71.0)$ \\
\hline \multicolumn{2}{|l|}{ Practicing exercise } \\
\hline Yes ( $1-5$ days/week, 30 min each) & $167(62.1)$ \\
\hline No & $102(37.9)$ \\
\hline \multicolumn{2}{|l|}{ Type of diabetes } \\
\hline Type 1 & $34(12.6)$ \\
\hline Type 2 & $235(87.4)$ \\
\hline \multicolumn{2}{|l|}{ Duration in years } \\
\hline$<10$ years & $115(42.8)$ \\
\hline$\geq 10$ years & $154(57.2)$ \\
\hline \multicolumn{2}{|l|}{ Frequency of testing } \\
\hline Glucose home testing at least once daily & $110(40.9)$ \\
\hline $\mathrm{HbA} 1 \mathrm{c}$, at least once every 3 months & $226(84.0)$ \\
\hline \multicolumn{2}{|l|}{${ }^{*} \mathrm{HbA} 1 \mathrm{C} \%$} \\
\hline Controlled, $\leq 6.5$ & $81(31.9)$ \\
\hline Uncontrolled, $>6.5$ & $173(68.1)$ \\
\hline \multicolumn{2}{|l|}{ *Blood pressure } \\
\hline High systolic blood pressure $>140 \mathrm{mmHg}$ & $88(33.0)$ \\
\hline High diastolic blood pressure $>90 \mathrm{mmHg}$ & $16(6.0)$ \\
\hline Combined, systolic > 140/diastolic > 90 mmHg & $98(36.7)$ \\
\hline \multicolumn{2}{|l|}{ Comorbidities } \\
\hline Hepatic & $67(24.9)$ \\
\hline Renal & $38(14.1)$ \\
\hline Cardiac & $32(11.9)$ \\
\hline Neurological & $15(5.6)$ \\
\hline Self-reported ophthalmic problems & $153(56.9)$ \\
\hline Self-reported numbness & $138(51.3)$ \\
\hline \multicolumn{2}{|l|}{ Diabetes distress } \\
\hline Distress of clinical importance, score $\geq 3$ & $33(12.3)$ \\
\hline Distress of any level, score $\geq 2$ & $104(38.7)$ \\
\hline \multicolumn{2}{|l|}{ *Lipid profile } \\
\hline LDL in mmol/L, median (IQR) & $2.45(1.04)$ \\
\hline High level of $L D L \geq 2.59 \mathrm{mmol} / \mathrm{L}$ & $118(44.2)$ \\
\hline Total cholesterol in mmol/L, median (IQR) & $4.27(1.36)$ \\
\hline High level of total cholesterol $\geq 5.18 \mathrm{mmol} / \mathrm{L}$ & $51(19.1)$ \\
\hline Triglycerides in $\mathrm{mmol} / \mathrm{L}$ & $1.39(0.73)$ \\
\hline High level of triglycerides $\geq 1.69 \mathrm{mmol} / \mathrm{L}$ & $70(26.2)$ \\
\hline $\mathrm{HDL}$ in $\mathrm{mmol} / \mathrm{L}$, median (IQR) & $1.14(0.4)$ \\
\hline Low level of $\mathrm{HDL} \leq 1.55 \mathrm{mmol} / \mathrm{L}$ & $227(85.0)$ \\
\hline
\end{tabular}

*Data were missing for two cases each in lipid profile and blood pressure, seven cases in $\mathrm{BMI}$, and in 15 cases in $\mathrm{HbA} 1 \mathrm{C}$ 
The mean value of the total cognitive score in relation to the duration of diabetes clearly showed an inverse significant association. This relation holds for the mean scores of the seven sub-domains but it was only significant in the alternating trial making and in the visuospatial/executive domain (Table 3). Figure 1 depicts the deficits in cognitive function by domain. Data show the percentage of participants who scored less than 60 out of the total 100 of the standardized score-of each subdomain. Overall, $80.3 \%$ of participants had cognitive impairment while $33.8 \%$ had severe impairment; meaning that $46.5 \%$ of participants scored 26-20 points. The domain of delayed recall was the worst as $80 \%$ of participants scored less than 60 out of 100 , followed by verbal fluency $(70 \%)$. Percentages of deficits were comparable for abstraction, alternating trial making and visuospatial/executive domain ranging from 43 to $52 \%$. The least affected domains were naming and scoring, $24 \%$ and $16 \%$ respectively.

We tested the association between level of cognitive impairment and sociodemographic and diabetes-related factors using the bivariate analysis: Table 4 shows that older age, low-level of education, low income, unemployment, long duration of diabetes, and DRD were significantly associated with cognitive impairment. Additionally, sedentary life and self-reported ophthalmic complications were significantly associated with severe cognitive impairment. Overall, the strength of association is small to moderate as indicated by the value of Cramer's V. Interestingly, other diabetes-related comorbidities did not show significant association with cognitive impairment (data not shown). Prevalence of CI was higher in patients with dyslipidemia, yet the difference did not reach a statistical significance. Also, the association between cognitive impairment and control of diabetes as defined by $\mathrm{HbA} 1 \mathrm{c} \leq 6.5 \%$ was not statistically significant.
Results of logistic regression analysis revealed that low level of education and self-reported ophthalmic complications were independently associated with severe cognitive impairment. Severe cognitive impairment was about six times more likely in less-educated participants, and two times more likely in patients with ophthalmic complications. Using the cutoff score $<26$ points, cognitive impairment was five-times more likely in less-educated participants, and two times more likely in patients with DRD. The association between the level of HbA1c and cognitive impairment did not reach a statistically significant level (Table 5); nevertheless, the probability of $\operatorname{cog}$ nitive impairment increased as the level of HbA1c increased, for example, at $\mathrm{HbA} 1 \mathrm{c}$ of $5 \%$, the probability of severe cognitive impairment was $29 \%$ versus $40 \%$ at HbA1c of $12 \%$ (Fig. 2).

\section{Discussion}

Our study demonstrated that more than $80 \%$ of participants had cognitive impairment. A study performed recently in Saudi Arabia [21] assessing cognitive function among patients aged 60 years and above reported that the prevalence of cognitive impairment was $46 \%$ based on the standard MoCA cutoff point. The difference between our results and these results regarding the MoCA score is mostly due to the selection criteria as our patients are all diabetics which is considered as a risk factor for development of cognitive impairment. Our results coincide with previous reports which suggest decline in global cognitive function and memory in participants with diabetes compared to those without diabetes $[7,13,31,32]$. The prevalence in our study is comparable to one study reported that the prevalence of $\mathrm{CI}$ among patients having diabetes with hemodialysis [33]. Another study reported further higher prevalence of $\mathrm{CI}$ among patients having diabetes with 87.5\% [34]. The lower prevalence reported in our study might be

Table 3 Mean score of total cognitive function and sub-domains according to duration of diabetes using the Montreal Cognitive Assessment scale

\begin{tabular}{|c|c|c|c|c|}
\hline \multirow[t]{2}{*}{ Scale/domains full scores } & \multicolumn{3}{|l|}{ Duration } & \multirow[t]{2}{*}{$P$} \\
\hline & $<10$ years, $n=115$ mean \pm SD & $10-19$ years, $n=81$ mean \pm SD & $\geq 20$ years, $n=73$ mean $\pm S D$ & \\
\hline Total (30) & $21.8 \pm 4.9$ & $22.1 \pm 4.9$ & $19.6 \pm 5.7$ & 0.004 \\
\hline \multicolumn{5}{|l|}{ Domains } \\
\hline Delayed recall (5) & $1.9 \pm 1.8$ & $2.2 \pm 1.7$ & $1.7 \pm 1.6$ & 0.137 \\
\hline Verbal fluency (3) & $1.9 \pm 0.98$ & $1.8 \pm 0.99$ & $1.6 \pm 0.88$ & 0.062 \\
\hline Abstraction (2) & $1.4 \pm 0.76$ & $1.3 \pm 0.72$ & $1.1 \pm 0.81$ & 0.07 \\
\hline Alternating trial making (6) & $4.1 \pm 1.7$ & $4.4 \pm 1.6$ & $3.7 \pm 1.9$ & 0.032 \\
\hline Visuoconstructional (5) & $3.6 \pm 1.4$ & $3.6 \pm 1.4$ & $2.9 \pm 1.7$ & 0.011 \\
\hline Naming (3) & $2.7 \pm 0.50$ & $2.7 \pm 0.54$ & $2.6 \pm 0.65$ & 0.249 \\
\hline Orientation (6) & $5.5 \pm 0.97$ & $5.6 \pm 0.87$ & $5.2 \pm 1.1$ & 0.43 \\
\hline
\end{tabular}




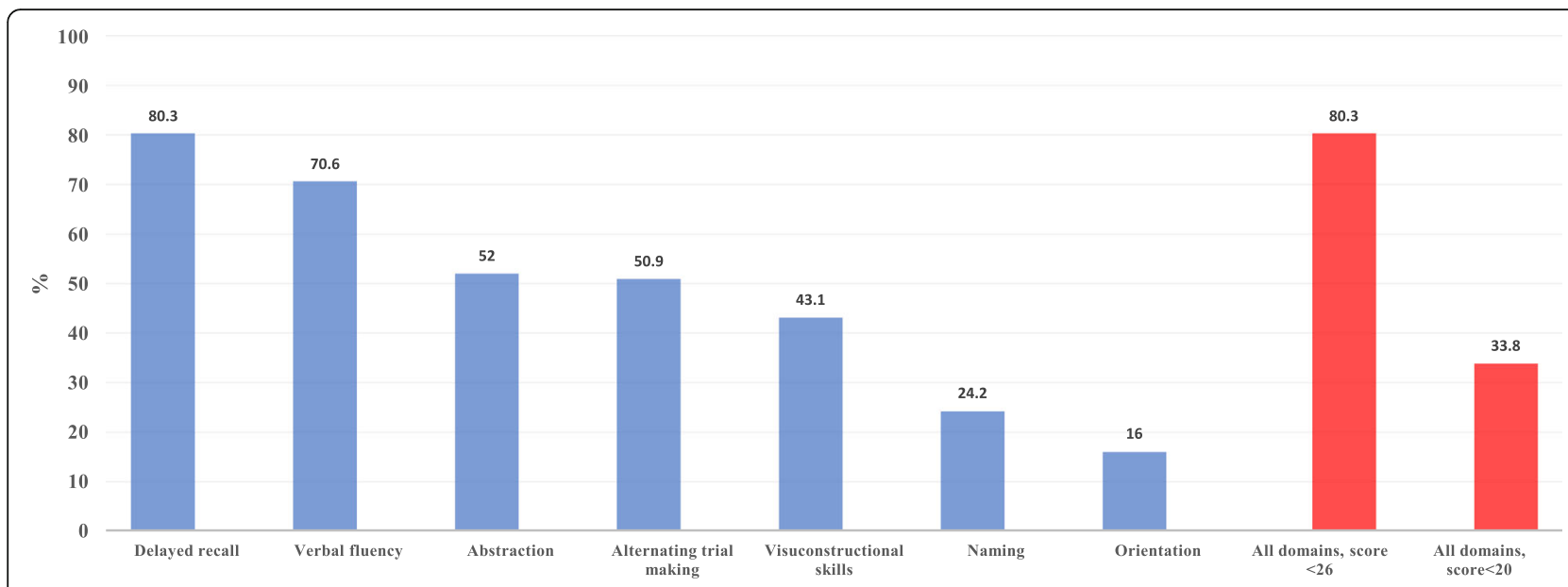

Fig. 1 Cognitive impairment by domain and in total among Saudi patients with diabetes using the Montreal Cognitive Assessment scale, $n=269$

explained by the additional decremental effect of end stage renal disease and hemodialysis on the cognitive function.

Our results demonstrated that more than one-third of the participants suffered from severe cognitive impairment. A study performed on younger age group stated that the prevalence of the cognitive impairment was $19.5 \%$ [16]. The difference might be attributed to the different age group selection in both studies and the more prevalence of risk factors of cognitive impairment in our patients. Another explanation for this difference is the diversity in the education level between the participants in both studies with lower level being in our population. The level of education has been considered as one of the most important determinants affecting MoCA score; in our study, education was independently associated with both severe and CI. It has been always suggested that one cutoff point is not ideal, especially for educationally diverse population [35].

Cognitive domains that have been studied included memory, psychomotor speed, visuospatial functions, frontal executive functions, processing speed, verbal fluency, and attention. Our results suggest that all cognitive domains are not affected equally. The domain of delayed recall was the worst affected followed by verbal fluency. Percentages of deficits were comparable for abstraction, alternating trial making and visuospatial/executive domains while the least affected domains were naming and scoring. In review of a literature on the association between diabetes and cognitive function conducted in India, the authors concluded that verbal memory and processing speed were the most domains affected with preservation of functions in other areas including visuospatial function, attention, semantic memory, and language [1]. These results are in line with our findings and was confirmed by another study which revealed impairment of executive function in diabetic individual at baseline and observed steeper declines in a test of language (phonemic verbal fluency) and executive function (Stroop color/word interference test) compared to individuals without diabetes. The results support previous findings of significant differences in executive function, language, and psychomotor speed/attention [36, 37]. One Japanese study recognized three types of cognitive impairment according to affected domain of MoCA score: frontal lobe impairment group in which the attention, language, and abstraction domains were affected, a temporal lobe impairment group with impaired recall domain, and the third is a mixed type [38]. Hishikawa et al. [39] found significant decrease of MoCA scores in "naming," "read list of letters," and "delayed recall" aspects. Similar results were reported by Zhong et al. [40]. Yuan and his colleague reported that elderly patients with hyperinsulinemia had lower cognitive test scores in MoCA, orientation, delayed memory, and attention/calculation domains [41].

The importance for identifying which cognitive domains are most affected by diabetes is to target the risk modification, possibly in the form of implementation of cognitive rehabilitation therapies including physical activity and exercise, which is needed to enhance executive functioning thus reducing the risk of cognitive impairment [13].

Several studies reported that the duration of diabetes is a key predictor of cognitive outcomes and that longstanding diabetes is associated with more significant cognitive impairment $[7,42]$. In our study, the mean value of the total cognitive score clearly showed an inverse significant association with the duration of diabetes. The relationship between the cognitive decline and the duration of diabetes may be due to the accumulating harmful effects which diabetes causes. Longer duration of diabetes increases the chances of hyperglycemic events which imposes an oxidative stress and causes 
Table 4 Frequency of cognitive impairment among patients with diabetes according to sociodemographic and disease variables

\begin{tabular}{|c|c|c|c|c|c|c|}
\hline \multirow[t]{2}{*}{ Variable } & \multicolumn{3}{|c|}{$\begin{array}{l}\text { Cognitive impairment (MOCA score } 26 \text { and } \\
\text { below) }\end{array}$} & \multicolumn{3}{|c|}{$\begin{array}{l}\text { Severe cognitive impairment (MOCA score } 20 \text { and } \\
\text { below) }\end{array}$} \\
\hline & Yes, $n$ (\%) & $P$ & Cramer's V & Yes, $n$ (\%) & $P$ & Cramer's V \\
\hline \multicolumn{7}{|l|}{ Age in years } \\
\hline$<40$ years & $10(55.6)$ & 0.001 & 0.22 & $1(5.6)$ & 0.008 & 0.19 \\
\hline 40-59 years & $105(76.6)$ & & & $43(31.4)$ & & \\
\hline$\geq 60$ years & $101(88.6)$ & & & $47(41.2)$ & & \\
\hline \multicolumn{7}{|l|}{ Gender } \\
\hline Females & $145(78.4)$ & 0.240 & & 70 (37.8) & 0.039 & 0.13 \\
\hline Males & $71(84.5)$ & & & $21(25.0)$ & & \\
\hline \multicolumn{7}{|l|}{ Level of education } \\
\hline Below bachelor & $149(90.3)$ & $<0.001$ & 0.32 & $81(49.09)$ & $<0.001$ & 0.41 \\
\hline Bachelor & $67(64.4)$ & & & $10(9.62)$ & & \\
\hline \multicolumn{7}{|l|}{ Income } \\
\hline Enough with saving & $23(82.1)$ & 0.026 & 0.16 & $6(21.4)$ & 0.022 & 0.17 \\
\hline Enough & $160(77.3)$ & & & $67(32.4)$ & & \\
\hline Not enough & $33(97.1)$ & & & $18(52.9)$ & & \\
\hline \multicolumn{7}{|l|}{ Employment } \\
\hline Employed & $57(69.5)$ & 0.003 & 0.18 & $17(20.7)$ & 0.003 & 0.18 \\
\hline Unemployed & $159(85.0)$ & & & 74 (39.6) & & \\
\hline \multicolumn{7}{|l|}{ Practicing exercise } \\
\hline Yes & $132(79)$ & 0.508 & & $47(28.1)$ & 0.012 & 0.15 \\
\hline No & $184(82.4)$ & & & $44(43.1)$ & & \\
\hline \multicolumn{7}{|l|}{ Duration in years } \\
\hline$<10$ years & $92(80.0)$ & 0.041 & 0.15 & $36(31.3)$ & 0.097 & \\
\hline 10-19years & $59(72.9)$ & & & $23(28.4)$ & & \\
\hline$\geq 20$ years & $65(89.0)$ & & & $32(43.8)$ & & \\
\hline \multicolumn{7}{|l|}{ Diabetic distress } \\
\hline Normal, score $<2$ & $124(75.2)$ & 0.008 & 0.16 & $53(32.1)$ & 0.456 & \\
\hline Any level, score $\geq 2$ & $92(88.5)$ & & & $38(36.5)$ & & \\
\hline \multicolumn{7}{|l|}{ Level of $\mathrm{HbA} 1 \mathrm{C}$} \\
\hline Controlled, $\leq 6.5 \mathrm{mmol} / \mathrm{L}$ & $66(81.5)$ & 0.671 & & $23(28.4)$ & 0.278 & \\
\hline Uncontrolled, $>6.5 \mathrm{mmol} / \mathrm{L}$ & $137(79.2)$ & & & $61(35.3)$ & & \\
\hline \multicolumn{7}{|l|}{ Dyslipidemia } \\
\hline Normal levels & $21(75.0)$ & 0.470 & & $9(32.1)$ & 0.853 & \\
\hline Abnormal levels & $193(80.8)$ & & & $81(33.9)$ & & \\
\hline \multicolumn{7}{|l|}{ Comorbidities } \\
\hline No ophthalmic problems & $89(76.7)$ & 0.200 & & $24(20.7)$ & $<0.001$ & 0.24 \\
\hline Yes (self-reported) & $127(83.0)$ & & & $67(43.8)$ & & \\
\hline
\end{tabular}

Cognitive impairment: measured by Montreal Cognitive Assessment scale (MoCA), total score equals 30; the score was adjusted for the low level of education by adding 1 point for an individual who has 12 years or fewer of formal education. The cutoff of cognitive impairment is $<26$ points, and the cutoff of severe cognitive impairment is < 20 points. Practicing exercise was considered for practicing 1-5 days/week, 30 min each. Dyslipidemia: high levels of LDL, TG, and low level of HDL. Other comorbidities did not show significant association with the cognitive impairment

glucose and glutamate toxicity, directly harming neurons. In addition, microvascular complications, including cerebral microangiopathy, occur with hyperglycemia and possibly increase the risk of cognitive impairment [19].
In this study, the inverse significant association with the duration of diabetes holds for the mean scores of the seven sub-domains, but it was only significant in the alternating trial making and in the visuospatial/executive 
Table 5 Logistic regression analysis of sociodemographic and diabetes-related variables in relation to cognitive impairment among patients with diabetes

\begin{tabular}{|c|c|c|c|c|}
\hline \multirow[t]{2}{*}{ Factor } & \multicolumn{2}{|c|}{ Cognitive impairment } & \multicolumn{2}{|c|}{ Severe cognitive impairment } \\
\hline & $\overline{\mathrm{OR}}$ & $95 \% \mathrm{Cl}$ & $\overline{\text { OR }}$ & $95 \% \mathrm{Cl}$ \\
\hline Older age category $\geq 50$ & 1.27 & $0.56-2.86$ & 1.46 & $0.60-3.52$ \\
\hline Female gender & 0.49 & $0.21-1.15$ & 1.06 & $0.49-2.30$ \\
\hline Low level of education & 4.67 & $2.11-10.33^{*}$ & 5.86 & $2.57-13.35^{*}$ \\
\hline Occupation (employed) & 0.58 & $0.27-1.26$ & 0.57 & $0.27-1.23$ \\
\hline Income (Low income) & 4.59 & $0.57-37.31$ & 1.34 & $0.56-3.21$ \\
\hline Not exercising & 1.00 & $0.47-2.09$ & 1.54 & $0.84-2.86$ \\
\hline Duration of diabetes & 1.23 & $0.74-1.70$ & 1.17 & $0.85-1.61$ \\
\hline Diabetes distress of any level & 2.26 & $1.04-4.89^{*}$ & 1.02 & $0.55-1.88$ \\
\hline Ophthalmic complication & 1.21 & $0.57-2.54$ & 2.19 & $1.14-4.19^{*}$ \\
\hline HbA1c level & 0.96 & $0.75-1.23$ & 1.05 & $0.86-1.27$ \\
\hline Dyslipidemia & 2.06 & $0.58-7.23$ & 1.29 & $0.41-4.07$ \\
\hline
\end{tabular}

Cognitive impairment: measured by Montreal Cognitive Assessment scale (MoCA), total score equals 30 ; the score was adjusted for the low level of education by adding 1 point for an individual who has 12 years or fewer of formal education. The cutoff of cognitive impairment is < 26 points, and the cutoff of severe cognitive impairment is < 20 points. Diabetes distress was measured by Arabic version of DDS-17. The cutoff for clinically significant DRD is > 2. Dyslipidemia: high levels of LDL, TG, and low level of HDL

${ }^{*} P<0.05$

domain. Two previous studies demonstrated that the duration of diabetes was associated with executive function domains of cognition $[19,43]$.

In the current study, low level of education was significantly associated with the degree of cognitive impairment. This finding is also supported by Prakash et al. [44] who demonstrated that the higher the educational level, the lesser the risk of cognitive impairment. Similarly, Yerrapragada et al. [1] showed that low level of education is associated with lower cognitive scores. However, the level of education did not significantly affect the cognitive function in young adult diabetic patients [16]. Our study showed that female gender was associated with more cognitive impairment. This finding is in line with a recent literature [1] in which the percentage of cognitive impairment in females was more than in males. This finding is also supported by the Salthouse study [45] which demonstrated that cognitive decline is more prevalent among females.

It has been suggested that cognitive impairment is mainly caused by arteriosclerosis at the arteriole level. Since the retina and brain have common developmental

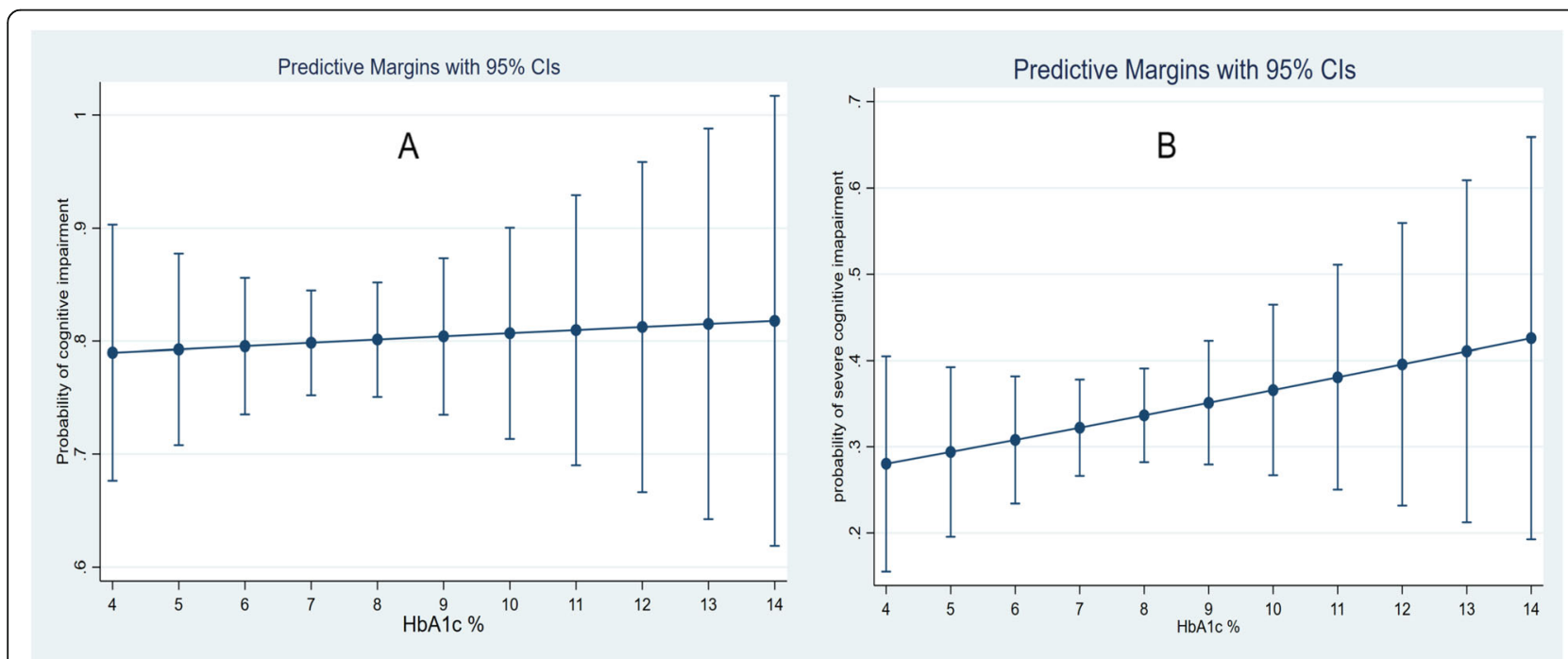

Fig. 2 Probability of cognitive impairment with $95 \%$ confidence interval in relation to HbA1c levels. a Cognitive impairment. b Sever cognitive impairment. The cutoff of cognitive impairment is $<26$ points, and the cutoff of severe cognitive impairment is $<20$ points 
origins and share anatomical, physiological, and autoregulational properties, the vascular structures of the small arteries of the retina and brain are similar $[19,46]$. In our patients, the presence of self-reported ophthalmic complications was significantly associated only with severe cognitive impairment. A recent study by Gupta et al. [47] stated that the degree of diabetic retinopathy specially at the more severe stages is associated with increased risk of developing cognitive impairment, independent of vision. Therefore, we think that diabetesrelated microangiopathy is facilitated in the brain in subjects with ophthalmopathy, which could explain the association between ophthalmic complications and cognitive impairment.

Analyzing HbA1c levels and cognitive impairment, our study showed that the mean level of HbA1c was significantly higher in patients with severe cognitive impairment, and the probability of $\mathrm{CI}$ and severe cognitive impairment increased as the level of HbA1c increased. However, the level of diabetic control using HbA1c cutoff value of $<6.5 \%$ was not independently associated with CI. Of note, control of diabetes was achieved in only one-third of participants, the small number of participants in the arm of controlled diabetes might affect the result, yet the direct relationship between the probability of $\mathrm{CI}$ and the level of CI should be considered clinically significant. Studies that found that HbA1c of $6.5 \%$ or above was associated with lower cognitive performance $[8,48-51]$. Surprisingly, a weak negative relationship between the HbA1c level and the cognitive function was detected by another study [16]. This inconsistency may be attributable to variations in the study design, study participants, duration or severity of diabetes, and the tools used to assess the degree of cognitive impairment.

Our study showed that the prevalence of CI was higher in patients with dyslipidemia, yet the difference did not reach a statistical significance. Over the previous years, studies have documented the significant contribution of dyslipidemia in the development of cognitive decline highlighting potential target for prevention [21, 52].

Finally, we found that minimal cognitive impairment was two times more likely among patients with diabeticrelated distress. This might be explained by the fact that the impact of distress on glycemic control is twofold or more which has a great effect on cognitive function [22].

We believe that these data can provide useful information from the clinical point of view. The high prevalence of cognitive impairment is of paramount clinical importance as patients with cognitive impairment may experience issues completing complex self-care practices, for example, self-care, independence, caloric intake calculation, regular blood sugar monitoring, insulin intake and dosage adjustment, and arranging meal plans and exercise schedules. Over time, poor self-management may be reflected as poor metabolic control and increment the danger of diabetes-related morbidities.

Some limitations of our study deserve consideration. First, it is a cross-sectional study, so causation cannot be inferred; also, convenient sampling technique would limit the generalizability of results. Second, we only have one reading of HbA1c for each participant, so we cannot assess trends in HbA1c levels. Third is lack of control group for assessing the effect of age per se on the cognitive function.

The strengths of our study include the following: first, this is the first study to assess CI in Saudi patients with diabetes. Second, we investigated the association between $\mathrm{CI}$ and diabetes-related variables including DRD. Third, the participation rate was satisfactory, and few data were missing. Lastly, the measurements of CI and DRD were reliable as evidenced by the value of the Cronbach's alpha.

\section{Conclusion}

From our study, we conclude that more than $80 \%$ of individuals with diabetes interviewed in our study had cognitive impairment and about one-third had severe impairment. The level of HbA1c was significantly higher in patients with severe cognitive impairment, and the probability of $\mathrm{CI}$ and severe cognitive impairment increased as the level of HbA1c increased. The total cognitive score was significantly lower with prolonged duration of diabetes. Low level of education, selfreported ophthalmic complications were associated with severe CI, while DRD and low level of education were associated with CI.

\section{Recommendations}

We recommend routine screening of cognitive function in older patients with DM for early detection and regular follow-up of cognitive impairment; hence, timely psychosocial interventions can be implemented to mitigate further deterioration in cognitive function. We also recommend conducting a prospective study on a larger sample size with multiple HbA1c readings and measuring the baseline cognitive impairment to compare the effect of duration of diabetes and fluctuation of blood sugar on cognitive function over time.

\section{Abbreviations}

Cl: Cognitive impairment; DM: Diabetes mellitus; DRD: Diabetes-related distress; MoCA: Montreal Cognitive Assessment scale; BMI: Body mass index; HbA1c: Hemoglobin A1c; BP: Blood pressure

Acknowledgements

We acknowledge the contribution of the patients in our study. 


\section{Authors' contributions}

$\mathrm{RN}, \mathrm{ES}$, and HE designed the study, analyzed and interpreted the patient data, and wrote the manuscript. FN, NA, AM, RA, KA, and BA collected the patients' data and applied the scales and performed literature search. All authors read and approved the final manuscript.

\section{Funding}

This research was funded by the Deanship of Scientific Research at Princess Nourah bint Abdulrahman University through the Fast-track Research Funding Program. Funding has no role in study design, data collection, data analysis, decision to publish or preparation of the manuscript.

\section{Availability of data and materials}

All data and materials used in the study are available for interested researchers upon approval from the Institutional Review Board at PNU. Contact IRB@pnu.edu.sa

\section{Ethics approval and consent to participate}

The study was conducted in accordance with the Helsinki Declaration and after the ethical approval of the Institutional Review Board at Princess Nourah bint Abdulrahman University, Riyadh, KSA (IRB-PNU:19-0139), date of approval 21 October 2019. Informed verbal consent was taken from all study participants. Verbal consent was approved by the ethics committee. The IRB recommends the use of verbal consent in survey procedures unless information is obtained in such a manner that human subjects can be identified or subjects' responses outside the research could place them at risk of criminal or civil liability or may damage their employability or reputation.

\section{Consent for publication}

Not applicable.

\section{Competing interests}

No conflict of interest.

\section{Author details}

'Clinical Science Department, College of Medicine, Princess Nourah bint Abdulrahman University, Riyadh, Kingdom of Saudi Arabia. ${ }^{2}$ Internal Medicine Department, Alexandria Faculty of Medicine, Alexandria University, Alexandria, Egypt. ${ }^{3}$ Psychiatry Department, Faculty of Medicine, Zagazig University, Zagazig, Egypt. ${ }^{4}$ High Institute of Public Health, Alexandria University, Alexandria, Egypt.

\section{Received: 14 July 2020 Accepted: 14 September 2020}

\section{Published online: 24 November 2020}

\section{References}

1. Yerrapragada DB, Rao CR, Karunakaran K, Lee HSE (2019) Cognitive dysfunction among adults with type 2 diabetes mellitus in Karnataka, India. Ochsner J 19(3):227-234

2. Yuan XY, Wang XG (2017) Mild cognitive impairment in type 2 diabetes mellitus and related risk factors: a review. Rev Neurosci 28(7):715-723

3. Cheng G, Huang $C$, Deng $H$, Wang $H$ (2012) Diabetes as a risk factor for dementia and mild cognitive impairment: a meta-analysis of longitudinal studies. Intern Med J 42(5):484-491

4. Yaffe K, Falvey C, Hamilton N, Schwartz AV, Simonsick EM, Satterfield S et al (2012) Diabetes, glucose control, and 9-year cognitive decline among older adults without dementia. Arch Neurol 69(9):1170-1175

5. Degen C, Toro P, Schonknecht P, Sattler C, Schroder J (2016) Diabetes mellitus type II and cognitive capacity in healthy aging, mild cognitive impairment and Alzheimer's disease. Psychiatry Res 240:42-46

6. Li W, Wang T, Xiao S (2016) Type 2 diabetes mellitus might be a risk factor for mild cognitive impairment progressing to Alzheimer's disease. Neuropsychiatr Dis Treat 12:2489-2495

7. Dybjer E, Nilsson PM, Engström G, Helmer C, Nägga K (2018) Prediabetes and diabetes are independently associated with adverse cognitive test results: a cross-sectional, population-based study. BMC Endocr Disord 18(1):91

8. Wium-Andersen IK, Rungby J, Jorgensen MB, Sandbaek A, Osler M, WiumAndersen MK (2019) Risk of dementia and cognitive dysfunction in individuals with diabetes or elevated blood glucose. Epidemiol Psychiatric Sci 29:e43

9. Kinoshita T, Shimoda M, Sanada J, Fushimi Y, Hirata Y, Irie S et al (2016) Association of GA/HbA1c ratio and cognitive impairment in subjects with type 2 diabetes mellitus. J Diabetes Complicat 30(8):1452-1455

10. Bordier L, Doucet J, Boudet J, Bauduceau B (2014) Update on cognitive decline and dementia in elderly patients with diabetes. Diabetes Metab 40(5):331-337

11. Mattishent K, Loke YK (2016) Bi-directional interaction between hypoglycaemia and cognitive impairment in elderly patients treated with glucose-lowering agents: a systematic review and meta-analysis. Diabetes Obes Metab 18(2):135-141

12. (2016) 2016 Alzheimer's disease facts and figures. Alzheimers Dement 12(4): 459-509

13. Palta P, Carlson MC, Crum RM, Colantuoni E, Sharrett AR, Yasar S et al (2017) Diabetes and cognitive decline in older adults: the ginkgo evaluation of memory study. J Gerontol A Biol Sci Med Sci 73(1):123-130

14. Alosco ML, Spitznagel MB, van Dulmen M, Raz N, Cohen R, Sweet LH et al (2012) Cognitive function and treatment adherence in older adults with heart failure. Psychosom Med 74(9):965-973

15. Ravona-Springer R, Schnaider-Beeri M (2011) The association of diabetes and dementia and possible implications for nondiabetic populations. Expert Rev Neurother 11(11):1609-1617

16. Roy S, Kim N, Desai A, Komaragiri M, Baxi N, Jassil N et al (2015) Cognitive function and control of type 2 diabetes mellitus in young adults. N Am J Med Sci 7(5):220-226

17. Umegaki H (2014) Type 2 diabetes as a risk factor for cognitive impairment: current insights. Clin Interv Aging 9:1011-1019

18. Marden JR, Mayeda ER, Tchetgen Tchetgen EJ, Kawachi I, Glymour MM (2017) High Hemoglobin A1c and diabetes predict memory decline in the health and retirement study. Alzheimer Dis Assoc Disord 31(1):48-54

19. West RK, Ravona-Springer R, Schmeidler J, Leroith D, Koifman K, GuerreroBerroa E et al (2014) The association of duration of type 2 diabetes with cognitive performance is modulated by long-term glycemic control. Am J Geriatr Psychiatry 22(10):1055-1059

20. Afgin $A E$, Massarwa $M$, Schechtman E, Israeli-Korn SD, Strugatsky R, Abuful A et al (2012) High prevalence of mild cognitive impairment and Alzheimer's disease in Arabic villages in northern Israel: impact of gender and education. J Alzheimers Dis 29(2):431-439

21. Alkhunizan M, Alkhenizan A, Basudan L (2018) Prevalence of mild cognitive impairment and dementia in Saudi Arabia: a community-based study. Dementia Geriatr Cogn Disord Extra 8(1):98-103

22. Asuzu CC, Walker RJ, Williams JS, Egede LE (2017) Pathways for the relationship between diabetes distress, depression, fatalism and glycemic control in adults with type 2 diabetes. J Diabetes Complicat 31 (1):169-174

23. Repple J, Karliczek G, Meinert S, Förster K, Grotegerd D, Goltermann J et al (2019) Variation of HbA1c affects cognition and white matter microstructure in healthy, young adults. Mol Psychiatry

24. Wermelt JA, Schunkert H (2017) Management of arterial hypertension. Herz. 42(5):515-526

25. Nasreddine ZS, Phillips NA, Bédirian V, Charbonneau S, Whitehead V, Collin I et al (2005) The Montreal Cognitive Assessment, MoCA: a brief screening tool for mild cognitive impairment. J Am Geriatr Soc 53(4):695-699

26. Ciesielska N, Sokołowski R, Mazur E, Podhorecka M, Polak-Szabela A, Kędziora-Kornatowska K (2016) Is the Montreal Cognitive Assessment (MoCA) test better suited than the Mini-Mental State Examination (MMSE) in mild cognitive impairment (MCl) detection among people aged over 60? Meta-analysis. Psychiatr Pol 50(5):1039-1052

27. Godefroy O, Fickl A, Roussel M, Auribault C, Bugnicourt JM, Lamy C et al (2011) Is the Montreal Cognitive Assessment superior to the Mini-Mental State Examination to detect poststroke cognitive impairment? A study with neuropsychological evaluation. Stroke. 42(6):1712-1716

28. Dong Y, Sharma VK, Chan BP, Venketasubramanian N, Teoh HL, Seet RC et al (2010) The Montreal Cognitive Assessment (MoCA) is superior to the Mini-Mental State Examination (MMSE) for the detection of vascular cognitive impairment after acute stroke. J Neurol Sci 299(1-2):15-18

29. Fisher L, Hessler DM, Polonsky WH, Mullan J (2012) When is diabetes distress clinically meaningful? Establishing cut points for the Diabetes Distress Scale. Diabetes Care 35(2):259-264

30. Polonsky WH, Fisher L, Earles J, Dudl RJ, Lees J, Mullan J et al (2005) Assessing psychosocial distress in diabetes: development of the diabetes distress scale. Diabetes Care 28(3):626-631 
31. Monette MC, Baird A, Jackson DL (2014) A meta-analysis of cognitive functioning in nondemented adults with type 2 diabetes mellitus. Can J Diabetes 38(6):401-408

32. Espeland MA, Miller ME, Goveas JS, Hogan PE, Coker LH, Williamson J et al (2011) Cognitive function and fine motor speed in older women with diabetes mellitus: results from the women's health initiative study of cognitive aging. J Womens Health (2002) 20(10):1435-1443

33. Cui L, Chen W, Yu X, Ju C (2020) The relationship between cognitive function and having diabetes in patients treated with hemodialysis. Int J Nursing Sci 7(1):60-65

34. Murray AM, Tupper DE, Knopman DS, Gilbertson DT, Pederson SL, Li S et al (2006) Cognitive impairment in hemodialysis patients is common. Neurology. 67(2):216-223

35. Lu J, Li D, Li F, Zhou A, Wang F, Zuo X et al (2011) Montreal cognitive assessment in detecting cognitive impairment in Chinese elderly individuals: a population-based study. J Geriatr Psychiatry Neurol 24(4):184190

36. Palta P, Schneider AL, Biessels GJ, Touradji P, Hill-Briggs F (2014) Magnitude of cognitive dysfunction in adults with type 2 diabetes: a meta-analysis of six cognitive domains and the most frequently reported neuropsychological tests within domains. J Int Neuropsychol Society 20(3):278-291

37. Spauwen PJ, Köhler S, Verhey FR, Stehouwer CD, van Boxtel MP (2013) Effects of type 2 diabetes on 12-year cognitive change: results from the Maastricht Aging Study. Diabetes Care 36(6):1554-1561

38. Mori Y, Futamura A, Murakami H, Kohashi K, Hirano T, Kawamura M (2015) Increased detection of mild cognitive impairment with type 2 diabetes mellitus using the Japanese version of the Montreal Cognitive Assessment: a pilot study. Neurol Clin Neurosci 3(3):89-93

39. Hishikawa N, Yamashita T, Deguchi K, Wada J, Shikata K, Makino H et al (2015) Cognitive and affective functions in diabetic patients associated with diabetes-related factors, white matter abnormality and aging. Eur J Neurol 22(2):313-321

40. Zhong Y, Miao Y, Jia WP, Yan H, Wang BY, Jin J (2012) Hyperinsulinemia, insulin resistance and cognitive decline in older cohort. Biomed Environ Sci 25(1):8-14

41. Zhao X, Han Q, Lv Y, Sun L, Gang X, Wang G (2018) Biomarkers for cognitive decline in patients with diabetes mellitus: evidence from clinical studies. Oncotarget. 9(7):7710-7726

42. Marseglia A, Fratiglioni L, Kalpouzos G, Wang R, Bäckman L, Xu W (2019) Prediabetes and diabetes accelerate cognitive decline and predict microvascular lesions: a population-based cohort study. Alzheimers Dement 15(1):25-33

43. Saczynski JS, Jónsdóttir MK, Garcia ME, Jonsson PV, Peila R, Eiriksdottir G et al (2008) Cognitive impairment: an increasingly important complication of type 2 diabetes: the age, gene/environment susceptibility--Reykjavik study. Am J Epidemiol 168(10):1132-1139

44. Prakash J, Ryali V, Srivastava K, Bhat PS, Shashikumar R (2011) Cognitive reserve: the warehouse within. Ind Psychiatry J 20(2):79-82

45. Salthouse TA (2009) When does age-related cognitive decline begin? Neurobiol Aging 30(4):507-514

46. Appaji A, Nagendra B, Chako DM, Padmanabha A, Hiremath CV, Jacob A et al (2019) Retinal vascular abnormalities in schizophrenia and bipolar disorder: a window to the brain. Bipolar Disord 21(7):634-641

47. Gupta P, Gan ATL, Man REK, Fenwick EK, Sabanayagam C, Mitchell P et al (2019) Association between diabetic retinopathy and incident cognitive impairment. Br J Ophthalmol 103(11):1605-1609

48. Rawlings AM, Sharrett AR, Schneider AL, Coresh J, Albert M, Couper D et al (2014) Diabetes in midlife and cognitive change over 20 years: a cohort study. Ann Intern Med 161(11):785-793

49. Biessels GJ, Staekenborg S, Brunner E, Brayne C, Scheltens P (2006) Risk of dementia in diabetes mellitus: a systematic review. Lancet Neurol 5(1):64-74

50. Cukierman-Yaffe T, Gerstein HC, Williamson JD, Lazar RM, Lovato L, Miller ME et al (2009) Relationship between baseline glycemic control and cognitive function in individuals with type 2 diabetes and other cardiovascular risk factors: the action to control cardiovascular risk in diabetes-memory in diabetes (ACCORD-MIND) trial. Diabetes Care 32(2): 221-226

51. Avadhani R, Fowler K, Barbato C, Thomas S, Wong W, Paul C et al (2015) Glycemia and cognitive function in metabolic syndrome and coronary heart disease. Am J Med 128(1):46-55
52. Lo Coco D, Lopez G, Corrao S (2016) Cognitive impairment and stroke in elderly patients. Vasc Health Risk Manag 12:105-116

\section{Publisher's Note}

Springer Nature remains neutral with regard to jurisdictional claims in published maps and institutional affiliations.

\section{Submit your manuscript to a SpringerOpen ${ }^{\circ}$ journal and benefit from:}

- Convenient online submission

- Rigorous peer review

- Open access: articles freely available online

- High visibility within the field

- Retaining the copyright to your article

Submit your next manuscript at $\boldsymbol{\nabla}$ springeropen.com 